\title{
Temperature-controlled fabrication of Co-Fe-based nanoframes for efficient oxygen evolution
}

\author{
Lvlv Ji ${ }^{1}$, Huifang Zheng ${ }^{1}$, Yujie Wei ${ }^{1}$, Shuaiqi Gong ${ }^{2}$, Tao Wang ${ }^{1}$, Sheng Wang ${ }^{{ }^{*}}$ and Zuofeng Chen ${ }^{2 *}$
}

\begin{abstract}
Transition metal phosphides (TMPs) have emerged as promising electrocatalysts to enhance the slow kinetic process of oxygen evolution reaction (OER). Framelike hollow nanostructures (nanoframes, NFs) provide the open structure with more accessible active sites and sufficient channels into the interior volume. Here, we report the fabrication of bimetallic Co-Fe phosphide NFs (Co-Fe-P NFs) via an intriguing temperature-controlled strategy for the preparation of precursors followed by phosphidation. The precursors, Co-Fe Prussian blue analogues (Co-Fe PBAs) are prepared by a precipitation method with $\mathrm{Co}^{2+}$ and $\left[\mathrm{Fe}(\mathrm{CN})_{6}\right]^{3-}$, which experience a structural conversion from nanocubes to NFs by increasing the aging temperature from 5 to $35^{\circ} \mathrm{C}$. The experimental results indicate that this conversion is attributable to the preferentially epitaxial growth on the edges and corners of nanocubes, triggered by intramolecular electron transfer at an elevated aging temperature. The as-prepared Co-Fe-P NFs catalyst shows remarkable catalytic activity toward OER with a low overpotential of $276 \mathrm{mV}$ to obtain a current density of $10 \mathrm{~mA} \mathrm{~cm}$, which is superior to the reference samples (Co-Fe-P nanocubes) and most of the recently reported TMPs-based electrocatalysts. The synthetic strategy can be extended to fabricate Co-Fe dichalcogenide NFs, thereby holding a great promise for the broad applications in energy storage and conversion systems.
\end{abstract}

Keywords: transition metal phosphides, nanoframes, oxygen evolution reaction, Prussian blue analogues, electrocatalysis

\section{INTRODUCTION}

The oxygen evolution reaction (OER) is an important half reaction for many energy storage and conversion systems, such as (photo)electrolytic water splitting, fuel cells and metal-air batteries [1-3]. Efficient catalysts are required to accelerate the reaction because of the sluggish OER kinetic process associated with $4 \mathrm{e}^{-} / 4 \mathrm{H}^{+}$transfer [4]. $\mathrm{IrO}_{2}$ and $\mathrm{RuO}_{2}$ are recognized as the state-of-the-art OER catalysts, but their large-scale applications are impeded by the high cost and earth scarcity of noble metals [5]. Therefore, extensive efforts are devoted to developing noblemetal-free OER catalysts.

To date, various inexpensive transition metal-based OER catalysts have been developed, including their oxide/(oxy) hydroxides, phosphides, chalcogenides, carbides, and nitrides [6-8]. In particular, transition metal phosphides (TMPs) have emerged as the promising candidates to catalyze OER efficiently due to their unique electronic structure, metallic conductivity and surface properties [9]. The post-catalysis analyses have revealed that the real active sites of TMPs are the in situ electrochemically oxidized, amorphous transition metal oxide/(oxy) hydroxides (TMOs) species on the surface, and the interior conductive TMPs provide rapid electron transfer channels for the surface catalyst layer, leading to synergistically enhanced OER performance [10].

To expose more accessible active sites, various nanostructured TMPs have been designed and fabricated, including nanowires, nanospheres, nanotubes, and nanosheets [11-14]. Among various nanostructures, the three-dimensional (3D) opened nanoframes (NFs) are of particular interest, which achieve large surface area and high atomic utilization efficiency by hollowing out the interior embedded unfunctional atoms [15]. Owing to the existence of abundant open channels, electrolyte can penetrate internally to create additional catalytic space and the produced gas bubbles can easily escape externally [16,17]. Despite their unique structural advantages, the examples of frame-like nanostructured TMPs are limited due to the lack of suitable synthetic strategy [18].

In addition to nanostructure engineering, the composition regulation, e.g., by rational design and synthesis of bimetallic phosphides, has also been recognized as an effective way to promote the catalytic activity [9]. Previous studies suggested that the redistribution of electrons can be induced by the strong interfacial interactions at the hybrid TMP heterointerface, leading to the improved electron conductivity and synergistic OER catalytic enhancement $[9,13,19]$. For instance, Liang et al. [20] reported that $\mathrm{Ni}_{2} \mathrm{P}-\mathrm{CoP}$ heterointerfaces can create effective electron transport channels, leading to synergistically enhanced OER performance with a low overpotential of $320 \mathrm{mV}$ to achieve a current density of $10 \mathrm{~mA} \mathrm{~cm}^{-2}$ in $1 \mathrm{~mol} \mathrm{~L}^{-1} \mathrm{KOH}$. Ren et al. [21] fabricated $\mathrm{Fe}_{2} \mathrm{P}-\mathrm{Ni}_{2} \mathrm{P}$ bimetallic phosphides, requiring a low overpotential of $318 \mathrm{mV}$ for $10 \mathrm{~mA} \mathrm{~cm}^{-2}$ in alkaline media.

Herein, we report the fabrication of $3 \mathrm{D}$ frame-like nanostructured cobalt-iron bimetallic phosphides (denoted as $\mathrm{Co}-\mathrm{Fe}$ P NFs) by a temperature-controlled strategy (Scheme 1). Framelike nanostructured cobalt-iron Prussian blue analogue precursors (denoted as Co-Fe PBA NFs) were firstly prepared by a simple precipitation method with $\mathrm{Co}^{2+}$ and $\left[\mathrm{Fe}(\mathrm{CN})_{6}\right]^{3-}$ at $35^{\circ} \mathrm{C}$, which were followed by phosphidation treatment at $350^{\circ} \mathrm{C}$ to obtain Co-Fe-P NFs. Interestingly, the Co-Fe PBA precursors experience a structural conversion from nanocubes to NFs by

\footnotetext{
${ }^{1}$ School of Materials Science and Engineering, Zhejiang Sci-Tech University, Hangzhou 310018, China

${ }^{2}$ School of Chemical Science and Engineering, Tongji University, Shanghai 200092, China

* Corresponding authors (emails: wangsheng571@hotmail.com (Wang S); zfchen@tongji.edu.cn (Chen Z))
} 

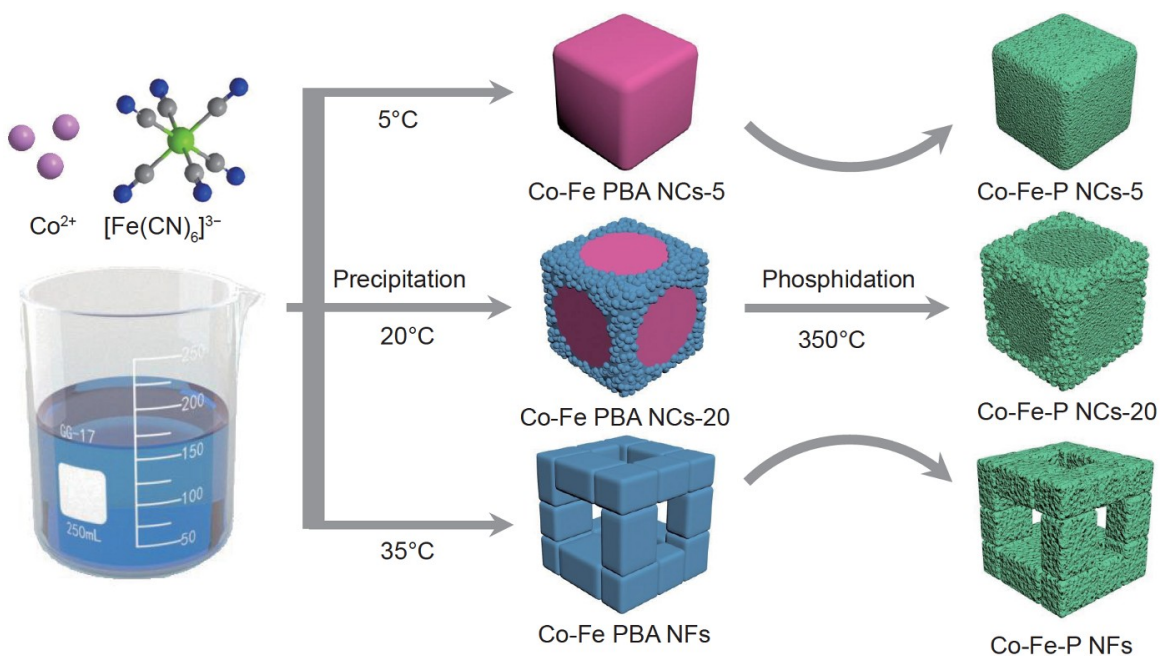

Scheme 1 Temperature-controlled synthesis of Co-Fe-P series samples. Co-Fe-Se and Co-Fe-S series samples can be obtained by selenylation and sulfuration treatments in the second step.

increasing the aging temperature from 5 to $35^{\circ} \mathrm{C}$, which is due to the preferentially epitaxial growth on the edges and corners of nanocubes, triggered by intramolecular electron transfer at an increased aging temperature. Benefiting from the unique framelike nanostructure and synergistic bimetallic composition, the resultant Co-Fe-P NF catalyst exhibits an excellent OER catalytic activity, achieving a current density of $10 \mathrm{~mA} \mathrm{~cm}^{-2}$ at a low overpotential of $276 \mathrm{mV}$ with a small Tafel slope of $35.8 \mathrm{mV} \mathrm{dec}^{-1}$ in $1 \mathrm{~mol} \mathrm{~L}^{-1} \mathrm{KOH}$. The OER catalytic performance of Co-Fe-P NFs is higher than that of the reference samples (CoFe-P nanocubes) and most of the recently reported TMPs-based electrocatalysts. The synthetic strategy can be extended to synthesize Co-Fe dichalcogenide NFs (Co-Fe-Se NFs and Co-Fe-S NFs), which also demonstrate competitive OER catalytic performances.

\section{EXPERIMENTAL SECTION}

\section{Reagents}

Cobalt acetate tetrahydrate $\left(\mathrm{Co}\left(\mathrm{CH}_{3} \mathrm{COO}\right)_{2} \cdot 4 \mathrm{H}_{2} \mathrm{O}, 99.5 \%\right)$, potassium hexacyanoferrate $\left(\mathrm{K}_{3}\left[\mathrm{Fe}(\mathrm{CN})_{6}\right], \geq 99.5 \%\right)$, trisodium citrate dihydrate $\left(\mathrm{Na}_{3} \mathrm{C}_{6} \mathrm{H}_{5} \mathrm{O}_{7} \cdot 2 \mathrm{H}_{2} \mathrm{O}, \geq 99 \%\right)$, sodium hypophosphite monohydrate $\left(\mathrm{NaH}_{2} \mathrm{PO}_{2} \cdot \mathrm{H}_{2} \mathrm{O}, 99 \%\right)$ and potassium hydroxide (KOH, 99.999\%) were purchased from Sigma-Aldrich Co. All other chemical reagents were of analytical grade and used as received without further purification. All electrolyte solutions were prepared with Milli-Qultrapure water $(\sim 18 \mathrm{M} \Omega \mathrm{cm})$.

\section{Apparatuses}

Fourier transform infrared (FT-IR) spectra were recorded on a Nicolet 6700 spectrometer (Thermo Fisher Nicolet, USA) with $\mathrm{KBr}$ pellets. Powder X-ray diffraction (XRD) patterns were recorded on a Rigaku D/max-2200 via ceramic monochromatized $\mathrm{Cu} K \alpha$ radiation $(\lambda=0.154 \mathrm{~nm})$, operating at $40 \mathrm{kV}$ and $40 \mathrm{~mA}$. Raman spectra were obtained on a confocal microscope laser Raman spectrometer (Rainshaw invia).

$\mathrm{X}$-ray photoelectron spectroscopy (XPS) for elemental analysis was conducted on a Kratos Axis Ultra DLD X-ray photoelectron spectrometer using $100-\mathrm{W}$ monochromatized $\mathrm{Al} \mathrm{Ka}$ radiation as the X-ray source for excitation. The $500-\mu \mathrm{m} X$-ray spot was used for XPS analysis. The base pressure in the analysis chamber was about $3 \times 10^{-10}$ mbar $(1 \mathrm{mbar}=100 \mathrm{~Pa})$. The $\mathrm{C} 1 \mathrm{~s}$ peak $(284.6 \mathrm{eV})$ was used for internal calibration. The peak resolution and fitting were processed with the XPS Peak 41 software.

The Brunauer-Emmett-Teller (BET) surface areas of the samples were measured on a Quanta Chrome Nova 2200e by nitrogen adsorption at $77.4 \mathrm{~K}$. The samples were degassed for $3 \mathrm{~h}$ at $300^{\circ} \mathrm{C}$ prior to measurements.

Scanning electron microscopy (SEM) images and energy dispersive X-ray (EDX) analysis data were obtained at ZEISS VLTRA-55 equipped with a Horiba EDX system. SEM images were obtained with an acceleration voltage of $3 \mathrm{kV}$ and EDX spectra were obtained with an acceleration voltage of $10 \mathrm{kV}$. Transmission electron microscopy (TEM) images, high-resolution TEM (HRTEM) images and selected area electron diffraction (SAED) patterns were obtained on a JEM-2010 HR.

Electrochemical measurements were conducted on a $\mathrm{CHI}$ 660E electrochemical workstation (Chenhua Corp., Shanghai, China).

\section{Catalysts preparation}

Synthesis of Co-Fe PBA NFs, Co-Fe PBA NCs-5 and Co-Fe PBA NCs-20 precursors

In a typical synthesis procedure, $0.15 \mathrm{~g} \mathrm{Co}\left(\mathrm{CH}_{3} \mathrm{COO}\right)_{2} \cdot 4 \mathrm{H}_{2} \mathrm{O}$ and $0.147 \mathrm{~g} \mathrm{Na}_{3} \mathrm{C}_{6} \mathrm{H}_{5} \mathrm{O}_{7} \cdot 2 \mathrm{H}_{2} \mathrm{O}$ were dissolved in $40 \mathrm{~mL}$ of water to form solution A. Meanwhile, $0.132 \mathrm{~g} \mathrm{~K}_{3}\left[\mathrm{Fe}(\mathrm{CN})_{6}\right]$ was dissolved in $60 \mathrm{~mL}$ of water to form solution $\mathrm{B}$. Then, solution $\mathrm{B}$ was added into solution A under magnetic stirring for $1 \mathrm{~min}$. The mixed solution was aged undisturbedly at $35^{\circ} \mathrm{C}$ for $36 \mathrm{~h}$. The precipitate was collected by centrifugation, washed with water and ethanol, and dried at $70^{\circ} \mathrm{C}$ overnight. In the parallel experiments, Co-Fe PBA NCs-5 and Co-Fe PBA NCs-20 were prepared under identical conditions except that the aging temperature was set to 5 and $20^{\circ} \mathrm{C}$, respectively.

Synthesis of Co-Fe-P NFs, Co-Fe-P NCs-5 and Co-Fe-P NCs-20 In a typical synthesis procedure, $10 \mathrm{mg} \mathrm{Co}-\mathrm{Fe} \mathrm{PBA} \mathrm{NFs}$ and $150 \mathrm{mg} \mathrm{NaH} \mathrm{PO}_{2} \cdot \mathrm{H}_{2} \mathrm{O}$ were put at two separate positions in a 
porcelain boat with $\mathrm{NaH}_{2} \mathrm{PO}_{2} \cdot \mathrm{H}_{2} \mathrm{O}$ at the upstream side of the tube furnace. The materials were heated at $350^{\circ} \mathrm{C}$ for $2 \mathrm{~h}$ in an $\mathrm{Ar}$ atmosphere at a ramping rate of $3^{\circ} \mathrm{C} \mathrm{min}$. In the parallel experiments, Co-Fe-P NCs-5 and Co-Fe-P NCs-20 were prepared under identical conditions except that the precursors were Co-Fe PBA NCs-5 and Co-Fe PBA NCs-20, respectively.

Synthesis of Co-Fe-Se NFs, Co-Fe-Se NCs-5 and Co-Fe-Se NCs-20 In a typical synthesis procedure, $10 \mathrm{mg}$ Co-Fe PBA NFs and $150 \mathrm{mg}$ Se powder were put at two separate positions in a porcelain boat with Se powder at the upstream side of the tube furnace. The materials were heated at $400^{\circ} \mathrm{C}$ for $2 \mathrm{~h}$ in an $\mathrm{Ar}$ atmosphere at a ramping rate of $3^{\circ} \mathrm{C} \mathrm{min}^{-1}$. In the parallel experiments, Co-Fe-Se NCs-5 and Co-Fe-Se NCs-20 were prepared under identical conditions except that the precursors were Co-Fe PBA NCs-5 and Co-Fe PBA NCs-20, respectively.

Synthesis of Co-Fe-S NFs, Co-Fe-S NCs-5 and Co-Fe-S NCs-20 In a typical synthesis procedure, $10 \mathrm{mg}$ Co-Fe PBA NFs and $150 \mathrm{mg} \mathrm{S}$ powder were put at two separate positions in a porcelain boat with $\mathrm{S}$ powder at the upstream side of the tube furnace. The materials were heated at $400^{\circ} \mathrm{C}$ for $2 \mathrm{~h}$ in an $\mathrm{Ar}$ atmosphere at a ramping rate of $3^{\circ} \mathrm{C} \mathrm{min}^{-1}$. In the parallel experiments, Co-Fe-S NCs-5 and Co-Fe-S NCs-20 were prepared under identical conditions except that the precursors were Co-Fe PBA NCs-5 and Co-Fe PBA NCs-20, respectively.

\section{Electrochemical measurements}

For the preparation of the working electrode, the electrocatalyst $(4 \mathrm{mg})$ and Nafion solution $(5 \mathrm{wt} \%, 80 \mu \mathrm{L})$ were dispersed in $1 \mathrm{~mL}$ of water/ethanol $(v / v, 4: 1)$ mixed solution. The electrocatalyst suspension $(5 \mu \mathrm{L})$ was dropped onto the glassy carbon electrode (diameter: $3 \mathrm{~mm}$ ), followed by drying at room temperature (mass loading: $\sim 0.265 \mathrm{mg} \mathrm{cm}^{-2}$ ). The three-electrode setup consisted of a working electrode, a carbon rod counter electrode, and a saturated calomel reference electrode (SCE). The potentials reported were referred to the reversible hydrogen electrode (RHE) via the Nernst equation: $E_{\mathrm{RHE}}=E_{\mathrm{SCE}}+0.059 \mathrm{pH}$ +0.244 . In $1 \mathrm{~mol} \mathrm{~L}^{-1} \mathrm{KOH}, E_{\mathrm{RHE}}=1.05 \mathrm{~V}+E_{\mathrm{SCE}}$. The OER catalytic performances of the electrocatalysts were evaluated in $\mathrm{N}_{2}$-saturated $1 \mathrm{~mol} \mathrm{~L}^{-1} \mathrm{KOH}$. Unless stated otherwise, linear sweep voltammetry (LSV) was conducted at a scan rate of $2 \mathrm{mV} \mathrm{s}^{-1}$. The Tafel slope was obtained from the LSV plot using a linear fit applied to points in the Tafel region. Electrochemical impedance spectroscopy (EIS) measurements were carried out from $10^{-2}$ to $10^{5} \mathrm{~Hz}$ with an amplitude of $5 \mathrm{mV}$ at different potentials. The electrochemical stability of the electrocatalyst was conducted by cyclic voltammetry (CV) scanning and longterm electrolysis. Unless stated otherwise, LSV and Tafel data plots were corrected with $90 \% i R$ compensation. The experiments were all performed at $22 \pm 2{ }^{\circ} \mathrm{C}$.

\section{RESULTS AND DISCUSSION}

\section{Synthesis and characterization of the Co-Fe-P electrocatalysts}

Fig. 1a-i show the SEM and TEM images of Co-Fe PBA series precursors prepared by precipitation of $\mathrm{Co}^{2+}$ and $\left[\mathrm{Fe}(\mathrm{CN})_{6}\right]^{3-}$ at different aging temperatures. At a low aging temperature of $5^{\circ} \mathrm{C}$, the precursor sample exhibits highly uniform nanocube structure with an average size of around $260 \mathrm{~nm}$ (Fig. 1a, d, g). When the aging temperature is elevated to $20^{\circ} \mathrm{C}$, small nanoparticles are preferentially grown on the edges and corners of the concave nanocubes (Fig. 1b, e, h). As the aging temperature further increases to $35^{\circ} \mathrm{C}$, the interior nanocubes disappear, while the exterior nanoparticles are further grown to nanocuboids, resulting in $3 \mathrm{D}$ opened frame-like nanostructures with an average size of around $500 \mathrm{~nm}$ (Fig. 1c, f, i).

XRD and FT-IR measurements were conducted to characterize Co-Fe PBA series precursors. As shown in Fig. 1j, XRD peaks of Co-Fe PBA NCs-5 at $17.3^{\circ}, 24.6^{\circ}, 35^{\circ}$ and $39.3^{\circ}$ are wellmatched with the cubic phase of $\mathrm{Co}_{3}\left[\mathrm{Fe}(\mathrm{CN})_{6}\right]_{2} \cdot x \mathrm{H}_{2} \mathrm{O}$ (JCPDS no. 46-0907) [22,23]. XRD pattern of Co-Fe PBA NCs-20 is still dominated by the characteristic peaks of $\mathrm{Co}_{3}\left[\mathrm{Fe}(\mathrm{CN})_{6}\right]_{2} \cdot x \mathrm{H}_{2} \mathrm{O}$, but three additional peaks at $17.9^{\circ}, 25.1^{\circ}$ and $36^{\circ}$ appear. These new peaks are indexed to a new Co-Fe PBA phase [24], in accordance with the epitaxially grown nanoparticles on the edges and corners of nanocubes. For Co-Fe PBA NFs, the characteristic peaks of $\mathrm{Co}_{3}\left[\mathrm{Fe}(\mathrm{CN})_{6}\right]_{2} \cdot x \mathrm{H}_{2} \mathrm{O}$ completely disappear and all peaks are ascribed to the new Co-Fe PBA phase. Such a structural transformation is also identified by FT-IR spectra (Fig. 1k, 1). With an increase of aging temperature, a decrease of the $v(\mathrm{~N} \equiv \mathrm{C})$ peak for $\mathrm{Co}^{\mathrm{II}}-\mathrm{N} \equiv \mathrm{C}-\mathrm{Fe}^{\mathrm{III}}$ at $2160 \mathrm{~cm}^{-1}$ is observed, which is accompanied with an emergence of a new strong $v(\mathrm{~N} \equiv \mathrm{C})$ peak for $\mathrm{Co}^{\mathrm{III}}-\mathrm{N} \equiv \mathrm{C}-\mathrm{Fe}^{\mathrm{II}}$ at $2123 \mathrm{~cm}^{-1}$ [25]. The previous study has revealed an inter-conversion between $\mathrm{Co}^{\mathrm{II}}-\mathrm{N} \equiv \mathrm{C}-\mathrm{Fe}^{\mathrm{III}}$ and $\mathrm{Co}^{\mathrm{III}}-\mathrm{N} \equiv \mathrm{C}-\mathrm{Fe}^{\mathrm{II}}$ blocks in Co-Fe PBAs, which is associated with intramolecular electron transfer from $\mathrm{Co}^{\mathrm{II}}$ to $\mathrm{Fe}^{\mathrm{III}}$ sites through the bridged cyano ligand (Scheme 2) [24].

During the precipitation synthesis, trisodium citrate are used as shape-directing surfactants, which selectively cap on $\{100\}$ facets of Co-Fe PBAs, leading to the formation of cube-like template with passivated side faces and highly active edges and corners [24]. Because Co-Fe PBA NCs and Co-Fe PBA NFs consist of $\mathrm{Co}^{\mathrm{II}}-\mathrm{N} \equiv \mathrm{C}-\mathrm{Fe}^{\mathrm{III}}$ and $\mathrm{Co}^{\mathrm{III}}-\mathrm{N} \equiv \mathrm{C}-\mathrm{Fe}^{\mathrm{II}}$ blocks, respectively, the morphology conversion is apparently associated with the intramolecular electron transfer. The process in the latter presumably leads to dissociation of nanocubes and the preferential epitaxial growth or re-assembly on the active edges and corners of nanocubes that form NFs. From our experimental results, the temperature-controlled morphologies indicate that the intramolecular electron transfer is restricted at a low aging temperature $\left(5^{\circ} \mathrm{C}\right)$, while it becomes pronounced at an elevated aging temperature $\left(35^{\circ} \mathrm{C}\right)$.

The Co-Fe PBA series precursors prepared by temperature regulation were converted to $\mathrm{Co}-\mathrm{Fe}-\mathrm{P}$ materials by phosphidation at $350^{\circ} \mathrm{C}$. The resultant Co-Fe-P NCs-5, Co-Fe-P NCs-20 and Co-Fe-P NFs were obtained by using Co-Fe PBA NCs-5, Co-Fe PBA NCs-20 and Co-Fe PBA NFs as precursors, respectively. Fig. 2a-f show the SEM and TEM images of Co-Fe-P series samples. Both nanocube and NF morphologies are wellmaintained after phosphidation treatment except that the surfaces become roughened. In particular, a large-range SEM image with numerous Co-Fe-P NFs is shown in Fig. S1, confirming the uniform NFs without aggregation. Meanwhile, a single NF is shown individually in Fig. 2g, which reveals small stacked nanoparticles in the framework with an average size of around $10 \mathrm{~nm}$. The HRTEM image of Co-Fe-P NFs reveals obvious lattice fringes with the interplanar spacings of around 0.2 and $0.24 \mathrm{~nm}$ (Fig. 2h and Fig. S2), which are attributable to the (112) and (111) crystal planes of CoP and FeP, respectively. In Fig. 2i, the SAED pattern exhibits three bright rings that arise from the 

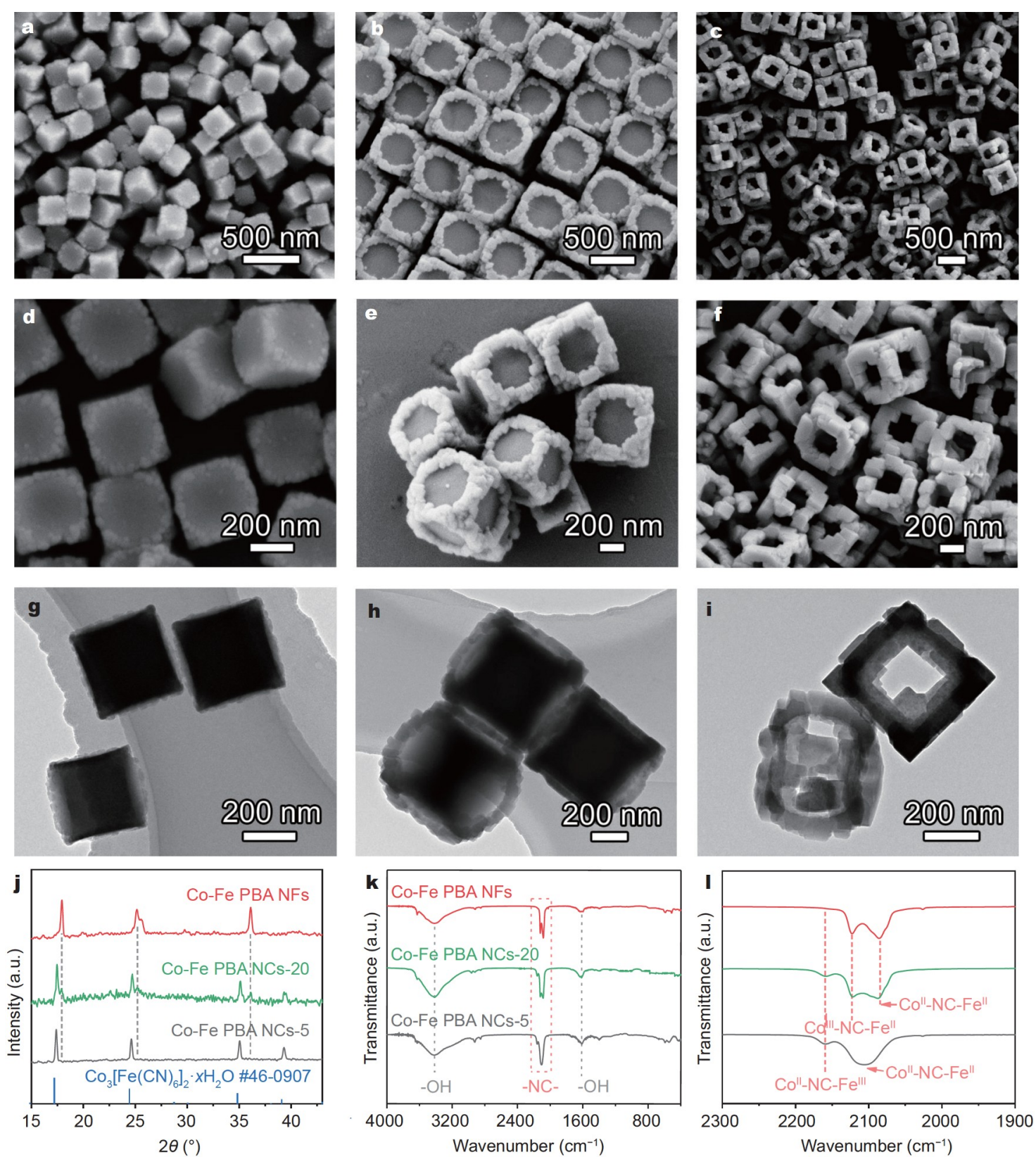

Figure 1 (a-f) SEM and (g-i) TEM images of (a, d, g) Co-Fe PBA NCs-5, (b, e, h) Co-Fe PBA NCs-20 and (c, f, i) Co-Fe PBA NFs, respectively. (j) XRD patterns, (k) FT-IR spectra and (l) magnified FT-IR spectra of Co-Fe PBA series precursors.

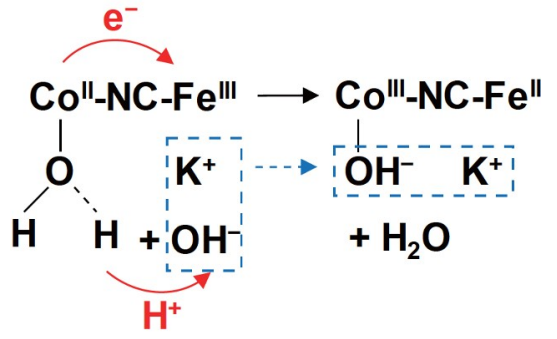

Scheme 2 Schematic illustration of intramolecular electron transfer from $\mathrm{Co}^{\mathrm{II}}$ to $\mathrm{Fe}^{\mathrm{III}}$ sites.

crystal planes of CoP (112) and FeP (111 and 011). Moreover, the EDX elemental mapping images display the uniform dis- tribution of $\mathrm{Co}, \mathrm{Fe}, \mathrm{P}, \mathrm{C}$ and $\mathrm{N}$ elements throughout the $\mathrm{NF}$ (Fig. 2j). The existence of $\mathrm{C}$ and $\mathrm{N}$ elements indicates the formation of $\mathrm{N}$-doped carbon matrix arising from pyrolysis of cyano ligands, which is also evidenced by Raman characterizations. In Fig. S3, two peaks at around 1353 and $1550 \mathrm{~cm}^{-1}$ can be observed for Co-Fe-P series samples, which are indexed to the D-band and G-band of carbon-based materials, respectively. The formation of $\mathrm{N}$-doped carbon matrix can avoid the overgrowth of $\mathrm{CoP}-\mathrm{FeP}$ nanoparticles and endow the catalyst with high conductivity and stability.

Fig. 3a shows the XRD patterns of the as-prepared Co-Fe-P series samples. As can be seen, the diffraction peaks for these samples are identical, which can be attributed to CoP (JCPDS no. 29-0497) and FeP (JCPDS no. 65-2595), confirming the conversion from Co-Fe PBAs to Co-Fe-P. The elemental com- 

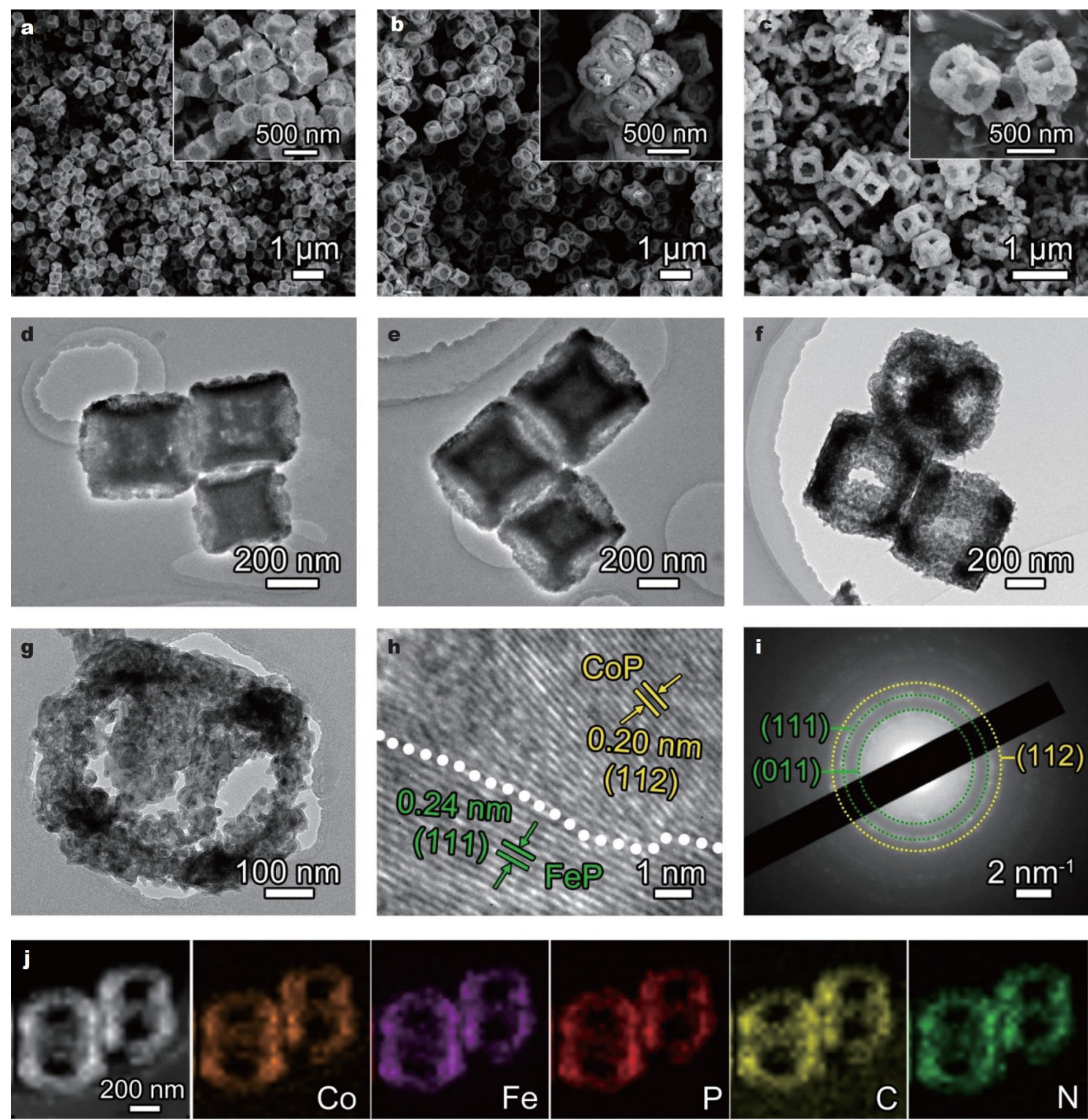

Figure 2 (a-c) SEM and (d-g) TEM images of Co-Fe-P samples: (a, d) Co-Fe-P NCs-5, (b, e) Co-Fe-P NCs-20, (c, f, g) Co-Fe-P NFs. (h) HRTEM image, (i) SAED pattern and (j) TEM-EDX elemental mapping images of Co-Fe-P NFs.

positions and electronic states of Co-Fe-P NFs, as a representative sample, were characterized by XPS. In Fig. S4a, the XPS survey spectrum of Co-Fe-P NFs shows the presence of $\mathrm{Co}, \mathrm{Fe}$, $\mathrm{P}, \mathrm{C}, \mathrm{N}$ and $\mathrm{O}$ elements, consistent with the result of EDX spectrum in Fig. S4b. Fig. $3 \mathrm{~b}$ shows the high-resolution XPS spectrum of Co 2p. The peaks at 778.6 and $793.8 \mathrm{eV}$ are attributable to the Co $2 \mathrm{p}_{3 / 2}$ and Co $2 \mathrm{p}_{1 / 2}$ peaks of $\mathrm{Co}-\mathrm{P}$ in $\mathrm{CoP}$, respectively [26]. The peaks at 781.7 and $797.5 \mathrm{eV}$ are ascribed to the surface-oxidized Co species and the peaks at 785.3 and $803.4 \mathrm{eV}$ are corresponding to the satellite peaks. For Fe 2p XPS in Fig. 3c, the peaks at 707.4 and $720.7 \mathrm{eV}$ are ascribed to the $\mathrm{Fe} 2 \mathrm{p}_{3 / 2}$ and $\mathrm{Fe} 2 \mathrm{p}_{1 / 2}$ peaks of $\mathrm{Fe}-\mathrm{P}$ in $\mathrm{FeP}$, while the peaks at 712.2 and $725.6 \mathrm{eV}$ are indexed to the surface-oxidized Fe species [27]. The P 2p XPS spectrum in Fig. 3d can be deconvoluted into three peaks. The doublet peaks at 129.4 and $130.4 \mathrm{eV}$ are assigned to $\mathrm{P} 2 \mathrm{p}_{3 / 2}$ and $\mathrm{P} 2 \mathrm{p}_{1 / 2}$ in CoP-FeP [28], while the peak at $133.5 \mathrm{eV}$ is ascribed to the surface oxidized $\mathrm{P}$ species. The $\mathrm{C} 1 \mathrm{~s}$
XPS spectrum in Fig. 3e can be deconvoluted into expected bonding states: $\mathrm{C}=\mathrm{C}(284.6 \mathrm{eV}), \mathrm{C}=\mathrm{N}(285.6 \mathrm{eV})$, and $\mathrm{O}-\mathrm{C}=\mathrm{O}$ $(288.5 \mathrm{eV})$. The $\mathrm{N} 1 \mathrm{~s}$ XPS spectrum in Fig. $3 \mathrm{f}$ can be deconvolved into three peaks: pyridinic $\mathrm{N}(398.1 \mathrm{eV})$, pyrrolic $\mathrm{N}(400.1 \mathrm{eV})$ and graphitic N $(401.4 \mathrm{eV})$. Together, the SEM, TEM, Raman, XRD and XPS results demonstrate the successful fabrication of Co-Fe-P NFs with CoP-FeP nanoparticles embedded in $\mathrm{N}$ doped carbon matrix.

\section{Electrocatalytic OER performance}

The OER catalytic performances of the as-prepared Co-Fe-P series samples were measured in $1 \mathrm{~mol} \mathrm{~L}^{-1} \mathrm{KOH}$ with a standard three-electrode setup. Before OER measurements, continuous $\mathrm{CV}$ scans were carried out in the potential range of $1-1.55 \mathrm{~V} v s$. RHE until a stable and reproducible CV curve was achieved. For comparison, commercial $\mathrm{RuO}_{2}$ was also examined under the identical test conditions. The LSV curves for the electrocatalysts 

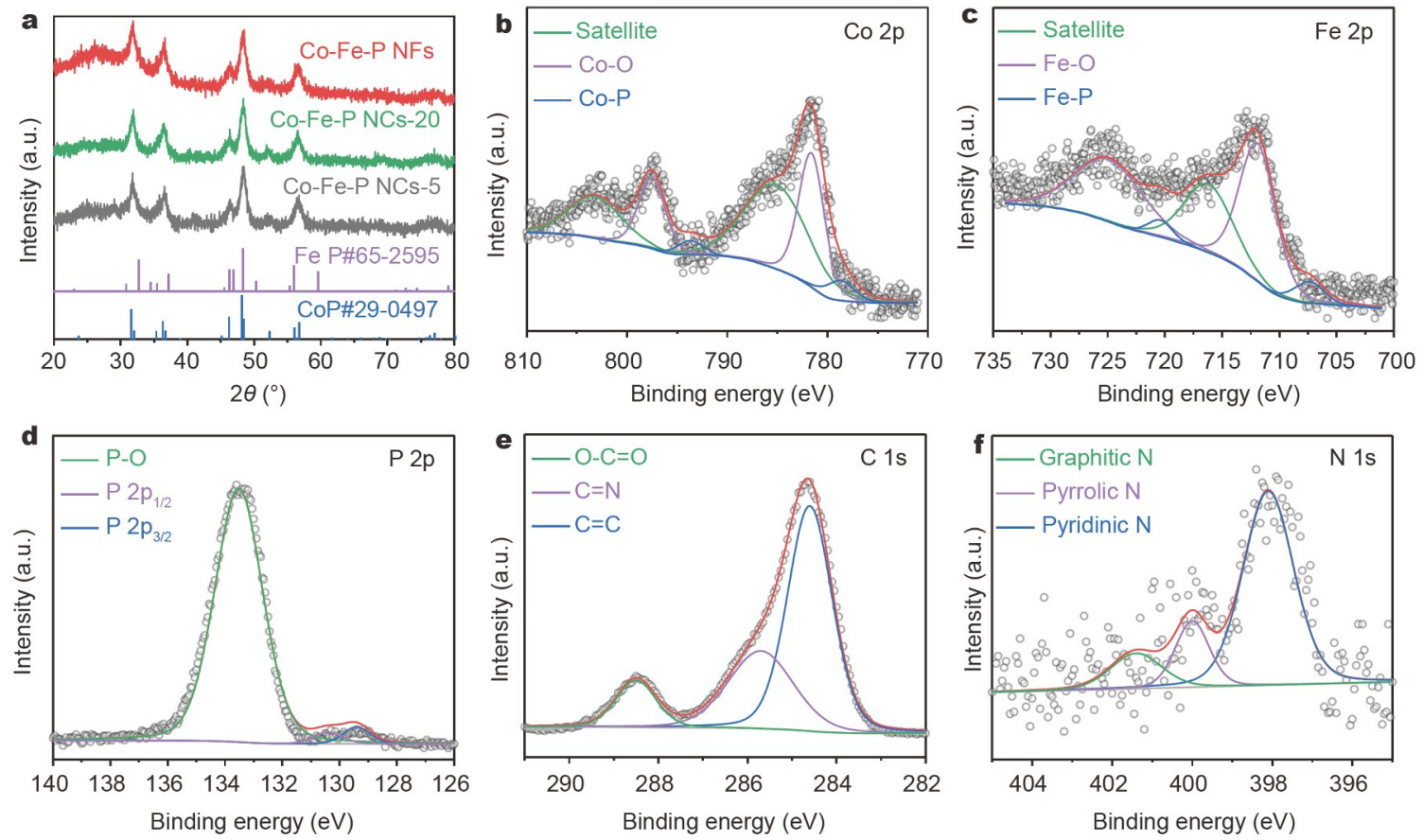

Figure 3 (a) XRD patterns of Co-Fe-P series samples. (b-f) High-resolution XPS spectra of Co-Fe-P NFs: (b) Co 2p, (c) Fe 2p, (d) P 2p, (e) C 1s and (f) N 1s.

are shown in Fig. 4a. For Co-Fe-P NFs, it requires a low overpotential of $276 \mathrm{mV}$ to deliver a current density of $10 \mathrm{~mA} \mathrm{~cm}^{-2}$, which is superior to that of Co-Fe-P NCs-20 $(290 \mathrm{mV})$ and CoFe-P NCs-5 (300 mV). Moreover, the OER catalytic performance of Co-Fe-P NFs is superior to that for $\mathrm{RuO}_{2}$ at increased overpotentials $(\eta>280 \mathrm{mV})$, although the latter exhibits an earlier catalytic onset. In previous studies, the OER performances of mono-metallic FeP $\left(\eta_{10}: 466 \mathrm{mV}\right)$ [29] and $\mathrm{CoP}\left(\eta_{10}: 354 \mathrm{mV}\right)$ [18] electrocatalysts prepared using corresponding Prussian blue nanocubes or their analogues as precursors, have been reported. Compared with these mono-metallic CoP or FeP electrocatalysts, the OER catalytic performances of bimetallic Co-Fe-P samples are greatly enhanced, indicating the synergistic enhancement effect by coupling CoP and FeP. In Fig. 4b, the corresponding Tafel plots show a lower Tafel slope for Co-Fe-P NFs $\left(35.8 \mathrm{mV} \mathrm{dec}^{-1}\right)$ than those for Co-Fe-P NCs-20 $\left(40.3 \mathrm{mV} \mathrm{dec}^{-1}\right)$ and Co-Fe-P NCs-5 (42.2 $\left.\mathrm{mV} \mathrm{dec}^{-1}\right)$. For a clear comparison, the OER catalytic performances of Co-Fe-P series samples are summarized in Table S1. The OER catalytic performance of Co-Fe-P NFs is also superior to most of the recently reported TMPs-based electrocatalysts as listed in Table S2.

To estimate the electrochemically active surface area (ECSA) of Co-Fe-P samples, the electrochemical double-layer capacitance $\left(C_{\mathrm{dl}}\right)$ was measured by $\mathrm{CV}$ scans at different scan rates within the potential window of $1.13-1.23 \mathrm{~V} v s$. RHE in $1 \mathrm{~mol} \mathrm{~L}^{-1}$ $\mathrm{KOH}$ (Fig. S5). As shown in Fig. 4c, the $C_{\mathrm{dl}}$ value of Co-Fe-P NFs is calculated as $13.9 \mathrm{mF} \mathrm{cm}^{-2}$, which is higher than that of Co-FeP NCs-20 (11.9 $\left.\mathrm{mF} \mathrm{cm}^{-2}\right)$ and Co-Fe-P NCs-5 $\left(7.3 \mathrm{mF} \mathrm{cm}^{-2}\right)$. In Fig. S6, the BET surface area of Co-Fe PBA NFs is $27 \mathrm{~m}^{2} \mathrm{~g}^{-1}$, which is also higher than that of Co-Fe-P NCs-20 $\left(20.3 \mathrm{~m}^{2} \mathrm{~g}^{-1}\right)$ and Co-Fe-P NCs-5 $\left(14.8 \mathrm{~m}^{2} \mathrm{~g}^{-1}\right)$. The comparisons of ECSA and BET specific surface areas of Co-Fe-P series samples confirm a higher dispersity and utilization of catalytic active sites with the unique frame-like nanostructure.
To make a comparison of the intrinsic catalytic activities for Co-Fe-P series samples, the LSV curves were normalized by the ECSA. As shown in Fig. 4d, the LSV curves of Co-Fe-P NCs-20 and $\mathrm{Co}-\mathrm{Fe}-\mathrm{P} \mathrm{NCs}-5$ are generally overlapped, while Co-Fe-P NFs exhibits an improved catalytic activity. The higher OER catalytic activity of Co-Fe-P NFs may be owing to the unique frame-like nanostructure with facilitated mass transfer and electrolyte penetration. EIS measurements were conducted to evaluate the reaction kinetics of Co-Fe-P samples. The Nyquist plots in Fig. 4e exhibit that Co-Fe-P NFs possesses a smaller charge transfer resistance than Co-Fe-P NCs-20 and Co-Fe-P $\mathrm{NCs}-5$ at an overpotential of $300 \mathrm{mV}$, which is in accordance with the higher catalytic activity of the former.

In Fig. 4f, the OER catalytic durability of Co-Fe-P NFs was examined by continuous CV scans from 1.1 to $1.55 \mathrm{~V} v s$. RHE and long-term electrolysis at an overpotential of $280 \mathrm{mV}$ in $1 \mathrm{~mol} \mathrm{~L}^{-1} \mathrm{KOH}$. Negligible catalytic degradation is observed after $3000 \mathrm{CV}$ cycles and the catalytic current density is sustainable after 25 -h electrolysis. These results indicate the excellent catalytic duribility for Co-Fe-P NFs.

After OER measurements in $1 \mathrm{~mol} \mathrm{~L}^{-1} \mathrm{KOH}$, the morphology, structure and surface composition of Co-Fe-P NFs were characterized by TEM, XRD and XPS. The TEM image of Co-Fe-P NFs shows the well-preserved NF morphology (Fig. S7) and $\mathrm{XRD}$ pattern of Co-Fe-P NFs exhibits the generally unaltered CoP-FeP crystalline phases (Fig. S8). Moreover, the Co $2 p$ and $\mathrm{Fe} 2 \mathrm{p}$ XPS spectra of Co-Fe-P NFs reveal predominant peaks of $\mathrm{CoOOH}$ and $\mathrm{FeOOH}$ on the surface (Fig. S9a, b). In Fig. S9c, the XPS peaks for $\mathrm{P} 2 \mathrm{p}$ almost disappeared, which was caused by the electrochemical oxidation of $\mathrm{P}$ to phosphates and their following dissolution into the electrolyte [10]. The surface electrochemical oxidation of $\mathrm{Co}-\mathrm{Fe}-\mathrm{P}$ to form a $\mathrm{Co}(\mathrm{Fe}) \mathrm{OOH}$ shell was also evidenced by $\mathrm{CV}$ scans in the potential range of $1-1.55 \mathrm{~V} v s$. $\mathrm{RHE}$ at $2 \mathrm{mV} \mathrm{s}^{-1}$ in $1 \mathrm{~mol} \mathrm{~L}^{-1} \mathrm{KOH}$. As shown in Fig. S10, a large 

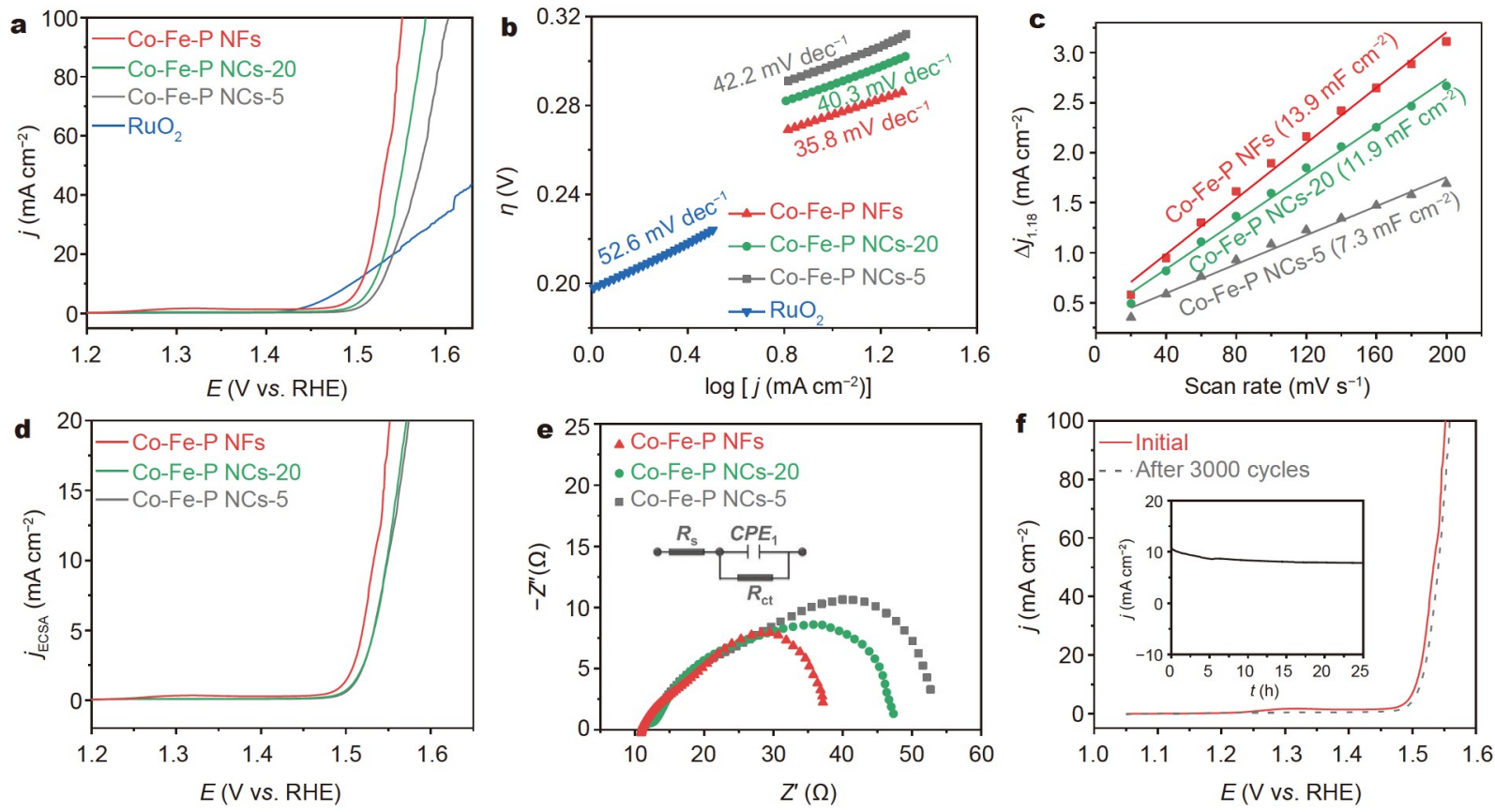

Figure 4 (a) LSV curves and (b) Tafel plots of Co-Fe-P series samples and commercial $\mathrm{RuO}_{2}$ for OER in $1 \mathrm{~mol} \mathrm{~L}^{-1} \mathrm{KOH}$. (c) The capacitive current at $1.18 \mathrm{~V}$ as a function of scan rate for Co-Fe-P series samples based on the CV results. (d) ECSA-normalized LSV curves of Co-Fe-P series samples. (e) Nyquist plots of Co-Fe-P series samples in $1 \mathrm{~mol} \mathrm{~L}^{-1} \mathrm{KOH}$ under an overpotential of $300 \mathrm{mV}$. The inset in (e) shows the equivalent electrical circuit used to model the EIS data, where $R_{\mathrm{s}}$ is the overall series resistance, $\mathrm{CPE}_{1}$ is the constant phase element and $R_{\mathrm{ct}}$ is the charge transfer resistance related to the OER electrocatalysis process. (f) LSV curves of Co-Fe-P NFs before and after $3000 \mathrm{CV}$ cycles. The inset in (f) shows the long-term electrolysis curve of Co-Fe-P NFs at an overpotential of $280 \mathrm{mV}$.

irreversible anodic wave at around $1.38 \mathrm{~V}$ can be observed in the first CV scan, which is attributable to the irreversible electrochemical oxidation of $\mathrm{Co}-\mathrm{Fe}-\mathrm{P}$ to $\mathrm{Co}(\mathrm{Fe}) \mathrm{OOH}$. This anodic wave is hardly observed in the second CV scan, indicating that the freshly formed $\mathrm{Co}(\mathrm{Fe}) \mathrm{OOH}$ shell can prevent further oxidation of internal Co-Fe-P sites. Together, the post OER characterizations indicate that an amorphous $\mathrm{Co}(\mathrm{Fe}) \mathrm{OOH}$ shell is in situ formed on the surface of $\mathrm{CoP}-\mathrm{FeP}$ nanoparticles during the OER process. The exterior amorphous $\mathrm{Co}(\mathrm{Fe}) \mathrm{OOH}$ is the catalytic active species for OER, while the interior conductive $\mathrm{Co}-\mathrm{Fe}$ $\mathrm{P}$ can ensure rapid electron transfer from/to the active shell.

The excellent OER catalytic performance of Co-Fe-P NFs can be ascribed to the following remarkable features. (i) The unique frame-like nanostructure is characterized with a high dispersity and utilization of catalytic active sites, which meanwhile is favorable for electrolyte penetration and gas bubbles emission. (ii) The N-doped carbon matrix in situ formed by the pyrolysis of cyano ligands is beneficial to obtaining highly dispersive CoPFeP nanoparticles with high conductivity and stability. (iii) The in situ electrochemically oxidized amorphous bimetallic $\mathrm{Co}(\mathrm{Fe}) \mathrm{OOH}$ shell serves as highly active sites for OER, while the interior CoP-FeP core of high conductivity can boost OER by providing rapid electron transfer channels.

\section{Extension to Co-Fe dichalcogenides NFs}

Transition metal chalcogenides have also been explored as OER electrocatalysts [30-34]. To demonstrate the versatility of the synthetic strategy, Co-Fe-Se NFs and Co-Fe-S NFs were synthesized by selenylation and sulfuration treatments of Co-Fe PBA NFs, respectively. For comparison, Co-Fe-Se NCs-5, Co$\mathrm{Fe}-\mathrm{Se} \mathrm{NCs}-20$, Co-Fe-S NCs-5 and Co-Fe-S NCs-20 were accordingly synthesized by using Co-Fe PBA NCs- 5 and Co-Fe
PBA NCs-20 as precursors. Fig. 5a-c show the SEM and TEM images of Co-Fe-Se series samples. After the selenylation treatment, the cube- or frame-like nanostructures are well-maintained with roughened surfaces. Despite different morphologies, these Co-Fe-Se series samples exhibit identical XRD patterns (Fig. 5d), consisting of $\mathrm{CoSe}_{2}$ (JCPDS no. 53-0449) and $\mathrm{FeSe}_{2}$ (JCPDS no. 74-0247). The HRTEM image of Co-Fe-Se NFs reveals lattice fringes with interplanar spacings of around 0.30 and $0.29 \mathrm{~nm}$ (Fig. 5e), which are ascribed to the crystal planes of $\mathrm{CoSe}_{2}$ (101) and $\mathrm{FeSe}_{2}$ (110), respectively. The corresponding SAED pattern (Fig. 5f) shows three bright rings that arise from the crystal planes of $\mathrm{CoSe}_{2}$ (101 and 111) and $\mathrm{FeSe}_{2}$ (011). Moreover, XPS spectra of Co-Fe-Se NFs are provided in Fig. S11, further substantiating the formation of Co-Fe-Se NFs. Similarly, SEM, TEM, XRD and XPS characterizations of Co-Fe-S series samples also demonstrate the successful conversion of Co-Fe PBA to Co-Fe-S consisting of heterostructured $\mathrm{CoS}_{2}$ and $\mathrm{FeS}_{2}$ after the sulfuration treatment (Fig. $5 \mathrm{~g}-1$ and Fig. S12).

The OER catalytic performances of $\mathrm{Co}-\mathrm{Fe}-\mathrm{Se}$ and $\mathrm{Co}-\mathrm{Fe}-\mathrm{S}$ series samples were also examined in $1 \mathrm{~mol} \mathrm{~L}^{-1} \mathrm{KOH}$. As shown in Fig. 6a, Co-Fe-Se NFs show higher catalytic activity $\left(\eta_{10}\right.$ : $303 \mathrm{mV})$ than Co-Fe-Se NCs-20 ( $\left.\eta_{10}: 319 \mathrm{mV}\right)$ and Co-Fe-Se NCs-5 $\left(\eta_{10}: 330 \mathrm{mV}\right)$. The corresponding Tafel plots also reveal superior OER catalytic kinetics for Co-Fe-Se NFs among Co-FeSe series samples (Fig. 6b). Similarly, the frame-like nanostructured samples Co-Fe-S NFs also show higher catalytic activity than cube-like nanostructured samples Co-Fe-S NCs-20 and Co-Fe-S NCs-5 (Fig. 6c, d). These results further corroborate the advantages of frame-like nanostructure by exposing more accessible catalytic active sites, reinforcing electrolyte penetration and facilitating the mass transfer. In addition, the long-term electrolysis measurements demonstrate the excellent 

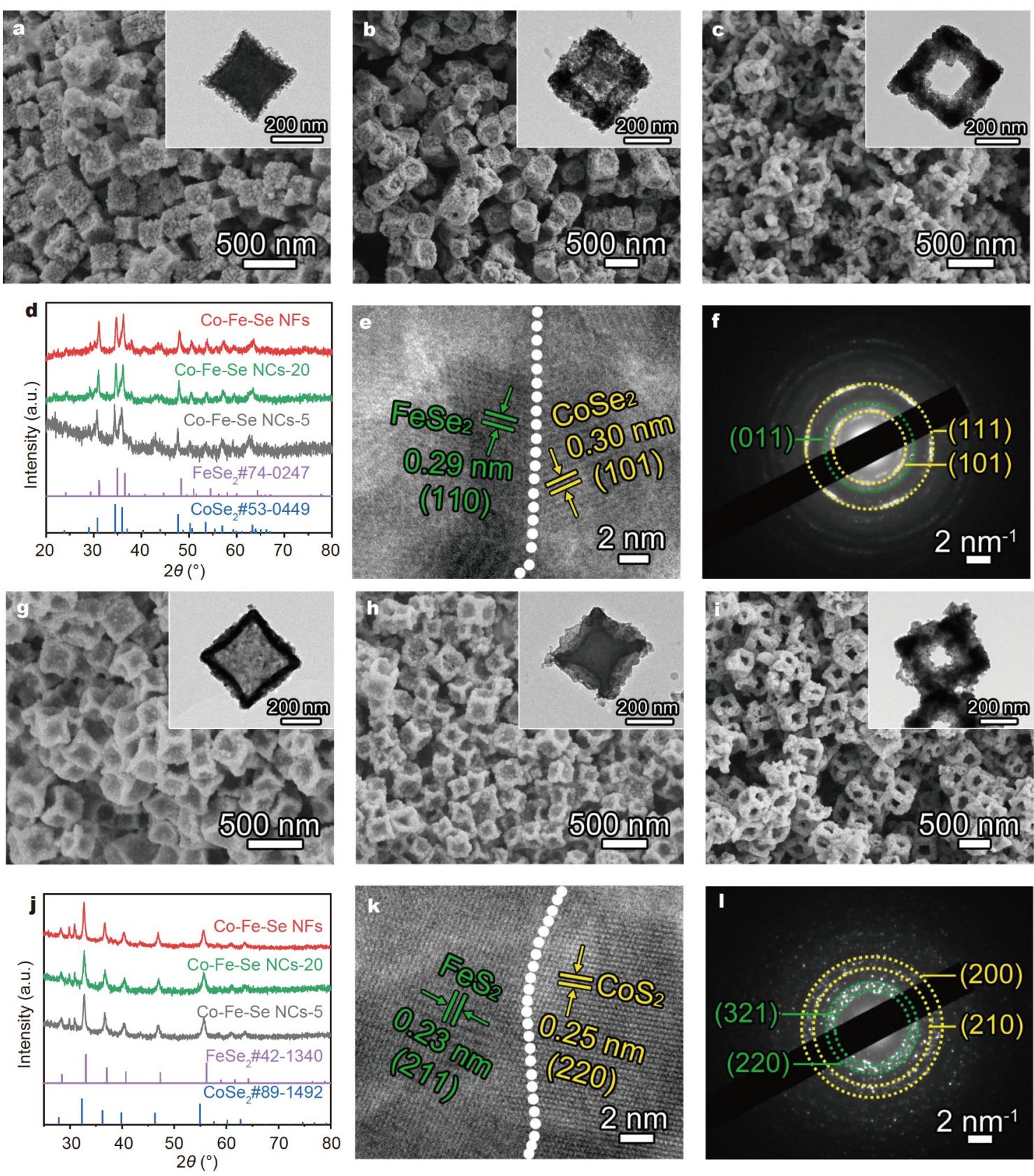

Figure 5 (a-c) SEM images and (d) XRD patterns of Co-Fe-Se NCs-5, Co-Fe-Se NCs-20 and Co-Fe-Se NFs. (e) HRTEM image and (f) SAED pattern of CoFe-Se NFs. The insets in $(\mathrm{a}-\mathrm{c})$ are the corresponding TEM images of Co-Fe-Se series samples. (g-i) SEM images and (j) XRD patterns of Co-Fe-S NCs-5, CoFe-S NCs-20 and Co-Fe-S NFs. (k) HRTEM image and (l) SAED pattern of Co-Fe-S NFs. The insets in (g-i) are the corresponding TEM images of Co-Fe-S series samples.

catalytic duribility for Co-Fe-Se NFs and Co-Fe-S NFs (insets in Fig. 6a, c). A summary of OER catalytic performances for Co-FeSe and Co-Fe-S series samples are supplemented in Fig. 6e and Table S1. As can be seen, the phosphidation product Co-Fe-P NFs exhibit relatively higher OER catalytic activity than Co-FeSe NFs and Co-Fe-S NFs, although they all have similar framelike nanostructures. This may be at least partially attributable to the relatively higher conductivity of TMPs for driving the faster electron transfer.

\section{CONCLUSIONS}

In summary, we report here the fabrication of frame-like nanostructured Co-Fe-P electrocatalyst via an interesting temperature-controlled strategy for the precursor preparation followed by phosphidation treatment. Effects of aging temperature on the morphology, structure and composition of Co-Fe PBA precursors were systematically investigated. The conversion from nanocubes to NFs is associated with the preferentially epitaxial growth on the edges and corners of nanocubes, induced by intramolecular electron transfer at an elevated aging temperature. Benefiting from the structural merits of NF and bimetallic phosphide composition, Co-Fe-P NFs exhibit remarkable cataltytic activity and stability toward OER. It requires a low overpotential of $276 \mathrm{mV}$ to reach a current density 

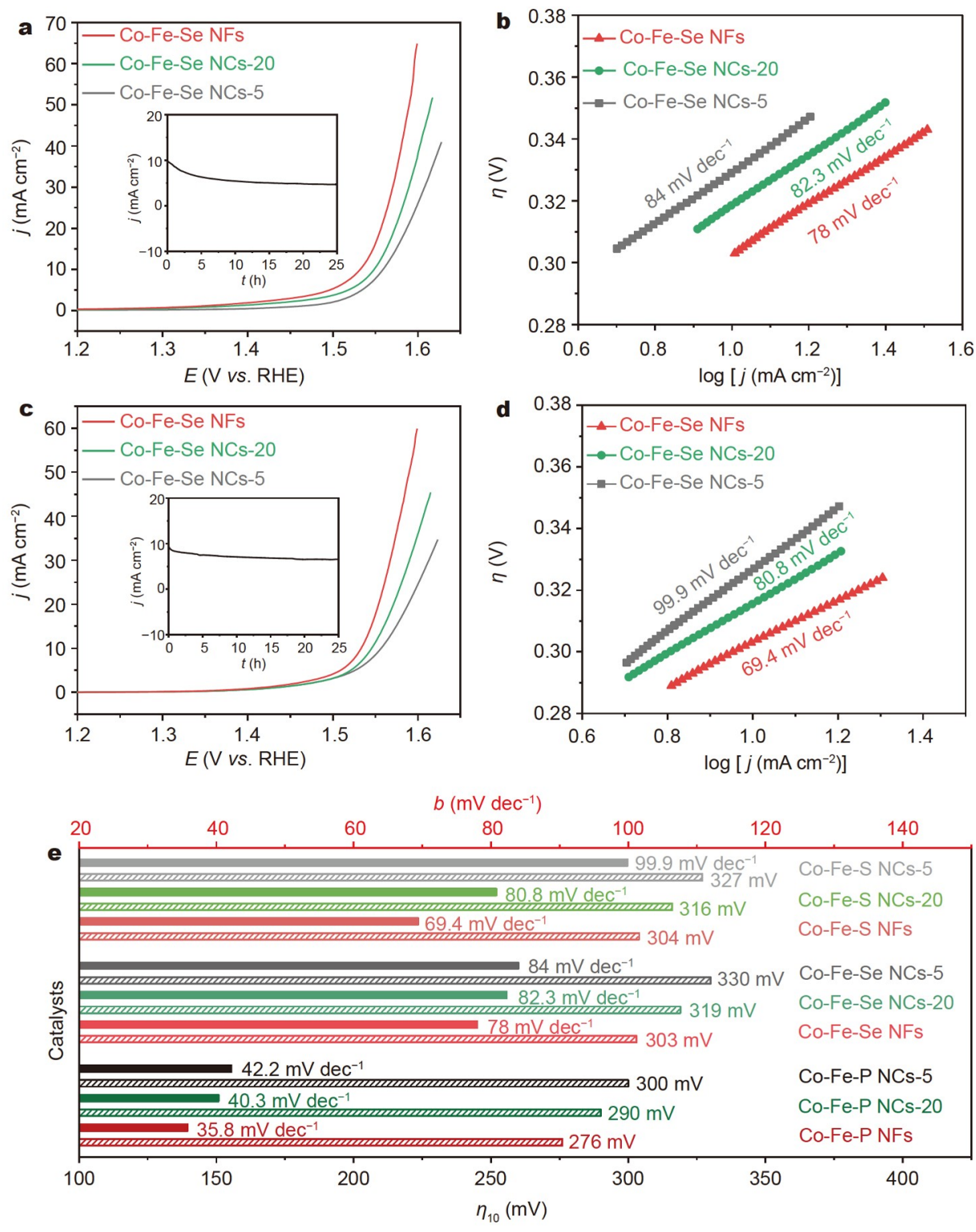

Figure 6 (a, c) LSV curves and (b, d) Tafel plots of (a, b) Co-Fe-Se and (c, d) Co-Fe-S series samples for OER in 1 mol L ${ }^{-1} \mathrm{KOH}$, respectively. Insets in (a, c) show the long-term electrolysis curves of (a) Co-Fe-Se NFs and (c) Co-Fe-S NFs at an overpotential of $300 \mathrm{mV}$, respectively. (e) A comparison of Co-Fe-P, CoFe-Se and Co-Fe-S series samples regarding the $\eta_{10}$ (twilled column) and Tafel slope ( $b$, solid column).

of $10 \mathrm{~mA} \mathrm{~cm}^{-2}$ in $1 \mathrm{~mol} \mathrm{~L}^{-1} \mathrm{KOH}$, outperfoming most of the recently reported TMPs-based electrocatalysts. The synthetic strategy can be extended to fabricate Co-Fe dichalcogenides NFs (Co-Fe-Se NFs and Co-Fe-S NFs), which also exhibit competitive OER catalytic performances. Considering the unique framelike nanostructure and synthetic accessibility, these Co-Fe-based NFs may find broad applications in the field of energy storage and conversion.

Received 24 April 2021; accepted 17 June 2021; published online 13 August 2021

1 Song J, Wei C, Huang ZF, et al. A review on fundamentals for designing oxygen evolution electrocatalysts. Chem Soc Rev, 2020, 49: 2196-2214

2 Wang HF, Chen L, Pang H, et al. MOF-derived electrocatalysts for oxygen reduction, oxygen evolution and hydrogen evolution reactions. Chem Soc Rev, 2020, 49: 1414-1448
3 Wu ZP, Lu XF, Zang SQ, et al. Non-noble-metal-based electrocatalysts toward the oxygen evolution reaction. Adv Funct Mater, 2020, 30: 1910274

4 Sun H, Yan Z, Liu F, et al. Self-supported transition-metal-based electrocatalysts for hydrogen and oxygen evolution. Adv Mater, 2020, 32: 1806326

5 Shi Q, Zhu C, Du D, et al. Robust noble metal-based electrocatalysts for oxygen evolution reaction. Chem Soc Rev, 2019, 48: 3181-3192

6 Lv L, Yang Z, Chen K, et al. 2D layered double hydroxides for oxygen evolution reaction: From fundamental design to application. Adv Energy Mater, 2019, 9: 1803358

7 Lyu F, Wang Q, Choi SM, et al. Noble-metal-free electrocatalysts for oxygen evolution. Small, 2019, 15: 1804201

8 Jamesh MI, Sun X. Recent progress on earth abundant electrocatalysts for oxygen evolution reaction (OER) in alkaline medium to achieve efficient water splitting-A review. J Power Sources, 2018, 400: 31-68

9 Li Y, Dong Z, Jiao L. Multifunctional transition metal-based phosphides in energy-related electrocatalysis. Adv Energy Mater, 2019, 10: 


\section{4}

10 Fang Z, Peng L, Qian Y, et al. Dual tuning of Ni-Co-A (A = P, Se, O) nanosheets by anion substitution and holey engineering for efficient hydrogen evolution. J Am Chem Soc, 2018, 140: 5241-5247

11 Bai Y, Zhang LC, Li Q, et al. Self-supported $\mathrm{CdP}_{2}-\mathrm{CDs}-\mathrm{CoP}$ for highperformance OER catalysts. ACS Sustain Chem Eng, 2021, 9: 12971303

12 Li N, Guan Y, Li Y, et al. Co-Mo-P carbon nanospheres derived from metal-organic frameworks as a high-performance electrocatalyst towards efficient water splitting. J Mater Chem A, 2021, 9: 1143-1149

13 Riyajuddin S, Azmi K, Pahuja M, et al. Super-hydrophilic hierarchical $\mathrm{Ni}$-foam-graphene-carbon nanotubes- $\mathrm{Ni}_{2} \mathrm{P}-\mathrm{CuP}_{2}$ nano-architecture as efficient electrocatalyst for overall water splitting. ACS Nano, 2021, 15: 5586-5599

14 Hou CC, Zou L, Wang Y, et al. MOF-mediated fabrication of a porous 3D superstructure of carbon nanosheets decorated with ultrafine cobalt phosphide nanoparticles for efficient electrocatalysis and zinc-air batteries. Angew Chem Int Ed, 2020, 59: 21360-21366

15 Fang Z, Wang Y, Liu C, et al. Rational design of metal nanoframes for catalysis and plasmonics. Small, 2015, 11: 2593-2605

16 Chen C, Kang Y, Huo Z, et al. Highly crystalline multimetallic nanoframes with three-dimensional electrocatalytic surfaces. Science, 2014, 343: 1339-1343

17 Cai ZX, Wang ZL, Xia YJ, et al. Tailored catalytic nanoframes from metal-organic frameworks by anisotropic surface modification and etching for the hydrogen evolution reaction. Angew Chem Int Ed, 2021, 60: $4747-4755$

18 Ji L, Wang J, Teng $\mathrm{X}$, et al. CoP nanoframes as bifunctional electrocatalysts for efficient overall water splitting. ACS Catal, 2020, 10: 412419

19 Zhang G, Li Y, Xiao X, et al. In situ anchoring polymetallic phosphide nanoparticles within porous Prussian blue analogue nanocages for boosting oxygen evolution catalysis. Nano Lett, 2021, 21: 3016-3025

20 Liang $\mathrm{X}$, Zheng $\mathrm{B}$, Chen $\mathrm{L}$, et al. MOF-derived formation of $\mathrm{Ni}_{2} \mathrm{P}-\mathrm{CoP}$ bimetallic phosphides with strong interfacial effect toward electrocatalytic water splitting. ACS Appl Mater Interfaces, 2017, 9: 23222 23229

21 Ren JT, Wang YS, Chen L, et al. Binary FeNi phosphides dispersed on $\mathrm{N}, \mathrm{P}$-doped carbon nanosheets for highly efficient overall water splitting and rechargeable Zn-air batteries. Chem Eng J, 2020, 389: 124408

22 Huang J, Xu P, Gao T, et al. Controlled synthesis of hollow bimetallic Prussian blue analog for conversion into efficient oxygen evolution electrocatalyst. ACS Sustain Chem Eng, 2020, 8: 1319-1328

23 Nai J, Wang S, Lou XWD. Ordered colloidal clusters constructed by nanocrystals with valence for efficient $\mathrm{CO}_{2}$ photoreduction. Sci Adv, 2019, 5: eaax5095

24 Nai J, Guan BY, Yu L, et al. Oriented assembly of anisotropic nanoparticles into frame-like superstructures. Sci Adv, 2017, 3: e1700732

25 Higel P, Villain F, Verdaguer M, et al. Solid-state magnetic switching triggered by proton-coupled electron-transfer assisted by long-distance proton-alkali cation transport. J Am Chem Soc, 2014, 136: 6231-6234

26 Pan Y, Sun K, Liu S, et al. Core-shell ZIF-8@ZIF-67-derived CoP nanoparticle-embedded $\mathrm{N}$-doped carbon nanotube hollow polyhedron for efficient overall water splitting. J Am Chem Soc, 2018, 140: 2610-2618

27 Zhao X, Zhang Z, Cao X, et al. Elucidating the sources of activity and stability of $\mathrm{FeP}$ electrocatalyst for hydrogen evolution reactions in acidic and alkaline media. Appl Catal B-Environ, 2020, 260: 118156

28 Niu Z, Qiu C, Jiang J, et al. Hierarchical CoP-FeP branched heterostructures for highly efficient electrocatalytic water splitting. ACS Sustain Chem Eng, 2018, 7: 2335-2342

29 Li Z, Zhao TT, Jiang WJ, et al. Bimetal Prussian blue as a continuously variable platform for investigating the composition-activity relationship of phosphides-based electrocatalysts for water oxidation. ACS Appl Mater Interfaces, 2018, 10: 35904-35910

30 Song W, Teng X, Liu Y, et al. Rational construction of self-supported triangle-like MOF-derived hollow $(\mathrm{Ni}, \mathrm{Co}) \mathrm{Se}_{2}$ arrays for electrocatalysis and supercapacitors. Nanoscale, 2019, 11: 6401-6409

31 Yang C, Zhang J, Gao G, et al. 3D metallic Ti@ $\mathrm{Ni}_{0.85} \mathrm{Se}$ with triple hierarchy as high-efficiency electrocatalyst for overall water splitting. ChemSusChem, 2019, 12: 2271-2277

32 Wang Z, Lin Z, Deng J, et al. Elevating the d-band center of sixcoordinated octahedrons in $\mathrm{Co}_{9} \mathrm{~S}_{8}$ through $\mathrm{Fe}$-incorporated topochemical deintercalation. Adv Energy Mater, 2020, 11: 2003023

33 Thangasamy P, Oh S, Nam S, et al. Ferrocene-incorporated cobalt sulfide nanoarchitecture for superior oxygen evolution reaction. Small, 2020, 16: 2001665

34 Li P, Zhao X, Duan X, et al. A multiphase nickel iron sulfide hybrid electrode for highly active oxygen evolution. Sci China Mater, 2020, 63: 356-363

Acknowledgements This work was supported by the National Natural Science Foundation of China (21872105 and 22072107) and the Natural Science Foundation of Zhejiang Province (LQ20B030001 and LY20E020002).

Author contributions Ji L designed this study, conducted the experiment and wrote the paper; Zheng $\mathrm{H}$ conducted the experiment and wrote the paper; Wei $Y$ and Gong S did some characterizations; Wang T performed some data analysis; Wang $\mathrm{S}$ and Chen $\mathrm{Z}$ designed this study and revised the paper. All authors contributed to the general discussion.

Conflict of interest The authors declare that they have no conflict of interest.

Supporting information Supporting data are available in the online version of the paper.

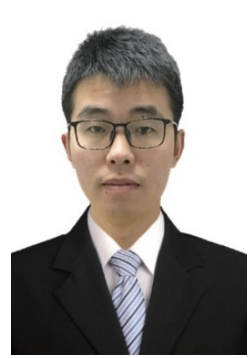

Lvlv Ji is currently a lecturer at Zhejiang Sci-Tech University. He received his BSc degree from Huainan Normal University in 2012, MSc degree from Zhejiang University of Technology in 2015, and $\mathrm{PhD}$ degree from Tongji University in 2018. His research interests focus on the synthesis and application of 3D complex hollow structured nanomaterials.

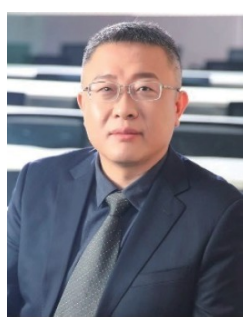

Sheng Wang received his $\mathrm{PhD}$ degree from Fukui University, Japan in 2003, and then worked as assistant lecturer at Hokkaido University for 3 years. He joined Zhejiang Sci-Tech University in 2006 and was promoted to a full professor in 2013. His research interests include (1) nano photocatalysis; (2) bio-inspired strategies for functional nanomaterials; and (3) micro/nano fibrous composites.

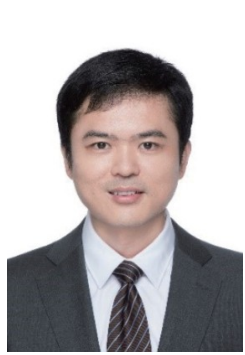

Zuofeng Chen got his PhD degree at the University of Hong Kong in 2009 and was a postdoctoral fellow at the University of North Carolina at Chapel Hill and Duke University. He has been a full professor at the School of Chemical Science and Engineering of Tongji University since 2014. His research interests focus on new energy materials and the electrocatalysts in energy conversion and storage systems. 


\section{温度调控合成钴铁基纳米框架用于高效电催化析氧} 反应

纪律律 ${ }^{1}$, 郑慧芳 ${ }^{1}$, 韦渝洁 ${ }^{1}$, 巩帅奇 ${ }^{2}$, 王駒金, 王戟 $1^{*}$, 陈作锋 $2^{*}$

摘要 过渡金属磷化物(TMPs)作为一类新型高效水分解电催化剂, 近 年来引起了研究人员的广泛兴趣. 框架型空心纳米结构通过去除内部 非功能性原子而具有独特的开放式结构, 可创造更多的内部反应空间, 提供丰富的活性位点和提升催化剂的原子利用率. 在本文中, 我们合成 了一种钴铁双金属磷化物纳米框架用于高效电催化析氧反应. 该催化 剂材料基于温度调控策略合成钴铁类普鲁士蓝纳米框架前驱体，随后 通过磷化处理制备. 实验发现, $\mathrm{Co}^{2+}$ 和 $\left[\mathrm{Fe}(\mathrm{CN})_{6}\right]^{3-}$ 共沉淀生成钴铁类普 鲁士蓝前驱体的过程中, 伴随反应温度从 $5^{\circ} \mathrm{C}$ 升高到 $35^{\circ} \mathrm{C}$, 产物结构由 实心纳米立方体转变为空心纳米框架. 研究表明, 纳米结构的转变与纳 米立方体棱角位置的选择性外延生长相关, 升高反应温度将触发前驱 体分子内的电子转移并诱导结构转变. 所制备的钴铁磷化物纳米框架 表现出优异的电催化析氧活性, 产生 $10 \mathrm{~mA} \mathrm{~cm}^{-2}$ 的催化电流密度仅需 $276 \mathrm{mV}$ 的过电位, 其性能优于对比样品 (钴铁磷化物实心纳米立方体) 和大部分近期报道的TMPs基电催化剂. 本文的合成策略可拓展制备钴 铁硫族化合物(如钴铁硒化物和钴铁硫化物)纳米框架, 因而在能量存储 与转化领域具有广泛的应用前景. 